Check for updates

Cite this: RSC Adv., 2019, 9, 28135

Received 26th July 2019

Accepted 31st August 2019

DOI: 10.1039/c9ra05818d

rsc.li/rsc-advances

\title{
Supramolecular structures of terbium(III) porphyrin double-decker complexes on a single-walled carbon nanotube surface $\uparrow$
}

\begin{abstract}
Ahmed I. A. Abd El-Mageed (D)*ab and Takuji Ogawa (D)*a
This work mainly reports the observation of novel supramolecular structures of Tb" ${ }^{\text {III }-5,15-}$ bisdodecylporphyrin (BDP, C12P) double-decker complexes on the surfaces of single-walled carbon nanotubes (SWNTs) performed by scanning tunneling microscopy under an ultra-high vacuum and low temperature, atomic force microscopy, scanning electron microscopy coupled with energy dispersive spectroscopy, and ultraviolet-visible spectroscopy. The molecules formed a well-ordered self-assembled helix-shaped array with regular periodicity on the tube surface. Additionally, some magnetic properties of the BDP-molecule as well as the resulting BDP-SWNT composites were investigated by superconducting quantum interference measurements. The molecule exhibits single-molecule magnetic (SMM) properties and the composite's magnetization increases almost linearly with decreasing temperature which is possibly due to the coupling between porphyrin molecules and SWNTs. Consequently, this may enable the development of more advanced spintronic devices based on porphyrin-nanocarbon composites.
\end{abstract}

\section{Introduction}

Owing to their importance and high applicability, carbon nanotubes (especially single-walled carbon nanotubes (SWNTs)) have become some of the most promising candidates in nanoscience and nanotechnology. They can be used in various applications, such as in molecular and nanoelectronics, ${ }^{1-3}$ sensing, ${ }^{4-7}$ and medicine ${ }^{8,9}$ and as catalysts. ${ }^{10,11}$ SWNTs have unusual properties compared to other materials. ${ }^{12}$ Hybridization of SWNTs with other molecules enables the emergence of new functionalities ${ }^{\mathbf{1 3 - 1 5}}$ via combination of the functionalities of the molecules and the high carrier mobility of SWNTs ${ }^{16-18}$ to afford important components for molecular-scale electronics. ${ }^{19-21}$ Another important property of SWNTs is magnetism, which is less studied than their electronic properties ${ }^{22-24}$ and can be important in relation to magnetic or spin-selective electronics.

Single-molecule magnets (SMMs) are single-molecular systems that exhibit slow magnetic relaxation below the blocking temperature $\left(T_{\mathrm{B}}\right){ }^{25-34}$ Hence, such molecules can work as memory devices for data storage. ${ }^{25,35}$ Since the first SMM model ${ }^{25}$ was developed $\left(\mathrm{Mn}_{12}\right.$ cluster complex with $\left.T_{\mathrm{B}}=3 \mathrm{~K}\right)$, in

${ }^{a}$ Chemistry Department, Graduate School of Science, Osaka University, Machikaneyama 1-1, Toyonaka, Osaka 560-0043, Japan. E-mail: abdelmageeda13@chem.sci.osaka-u.ac.jp; Ogawa@chem.sci.osaka-u.ac.jp ${ }^{b}$ Chemistry Department, Faculty of Science, Minia University, Minia 61519, Egypt † Electronic supplementary information (ESI) available. See DOI: 10.1039/c9ra05818d which the design strategy was to fabricate a polymetallic transition-metal complex having strong intramolecular exchange coupling with large anisotropy, SMMs have become promising candidates as effective units for applications in molecular spintronics (technology in which the spin coupling and molecular charge emerge in electronic devices). ${ }^{36}$ Later, a new category of lanthanide-based SMMs with phthalocyanine and/or porphyrin ligands was discovered,,$^{29,31,34,37-44}$ exhibiting slow magnetic relaxation due to the extremely high easy-axis magnetic anisotropy arising from the lanthanide ions and ligands (the electronic spin number $J$ of a $\mathrm{Tb}$ ion is 6 , and an unpaired electron is delocalized over the organic part). In particular, porphyrin/phthalocyanine-based $\mathrm{Tb}^{\mathrm{III}}$ double-decker complexes have attracted considerable attention, ${ }^{37-39,45-50}$ as they demonstrate higher blocking temperatures (i.e., 40, 50, and $54 \mathrm{~K})^{37-39}$ with large hysteresis loops as well as unique spectroscopic and redox properties originating from the strong $\pi-\pi$ interaction between the two ligands. ${ }^{51,52}$

$\mathrm{Tb}^{\mathrm{III}}$-porphyrin double-decker complexes have an important feature: they are present in three forms, i.e., protonated, anionic, and radical forms (Schemes 1 and $\mathrm{S} 1 \dagger$ ), and the electronic and magnetic properties of these forms can be switched as reversible interconversion between these forms is possible. ${ }^{48,51}$

Although the supramolecular structures of lanthanidetetraazolic-based double-decker complexes on flat nanocarbon surfaces, i.e., HOPG, have been extensively studied, ${ }^{45,46,53-58}$ the self-assembly of arrays on the curved carbon surfaces of SWNTs has not been reported yet, and it remains difficult to predict and 
understand the structures. However, some attempts have been made to reveal the chemistry needed to bridge the carbon nanotubes and double-decker domains. ${ }^{59-64}$

Previously, our group observed the self-assembly of a $\mathrm{Tb}^{\mathrm{III}}$ 5,15-bisdodecylporphyrin ( $\mathrm{Tb}^{\mathrm{III}}$-BDP) double-decker complex (protonated form) on the surface of $\mathrm{HOPG}^{\mathbf{4 6}}$ under ambient conditions using STM, where BDP forms distinctive lamellar structures separated by long ( $n$-dodecyl) chains, which not only stabilize the surface self-assembly, but also serve as physical spacers between the $\mathrm{Tb}$ sites. Additionally, we successfully assigned the absolute handedness chirality of SWNTs experimentally, for the first time, using BDP (C12P) ligand supramolecular structures and STM. ${ }^{65}$

Specifically, the objectives of this research were to investigate the supramolecular structures of the $\mathrm{Tb}^{\mathrm{III}}$-porphyrin doubledecker complex-SWNT composites using various microscopic characterization techniques, i.e., STM, atomic force microscopy (AFM), and scanning electron microscopy coupled with energy dispersive spectroscopy (SEM-EDS) and spectroscopic (i.e., UVvis). Consequently, in this study, for the first time, we successfully observed the supramolecular structures of $\mathrm{Tb}^{\mathrm{III}}$-porphyrin double-decker complex (i.e., $\mathrm{Tb}^{\mathrm{III}}-5,15$-bisdodecylporphyrin $\left(\mathrm{Tb}^{\mathrm{III}}\right.$-BDP) $)$ on the surfaces of SWNTs using the common types of scanning probe microscopy (SPM), i.e., STM and AFM. The topographic surfaces of the BDP-SWNT composites could be recognized using AFM, while the detailed structures of the BDP molecules could be observed on the tube surfaces using the atomic-resolution imaging capabilities of STM. Additionally, the resulting composites were characterized by performing ultraviolet-visible (UV-vis) spectroscopic measurements, which revealed Doppler shifts of the BDP spectra after complexation with SWNT. Moreover, some magnetic properties of the composites were studied and they interestingly showed butterfly-shaped hysteresis loops larger than that of the molecule alone as well as the composite's magnetization increases almost linearly with decreasing temperature. To the best of our knowledge, this is the first report to describe in detail the supramolecular structures of $\mathrm{Tb}^{\mathrm{III}}$-porphyrin double-decker complexes on the surfaces of SWNTs using SPM imaging techniques. The optimized structure of $\left[\mathrm{Y}(\mathrm{BDP})_{2}\right]^{\bullet}$ calculated using density functional theory (DFT) with B3LYP/3-21G (for C, H, and $\mathrm{N}$ ) and SDD (for Y) functional levels as well as the frontier molecular orbitals (highest occupied molecular orbital (HOMO) and lowest unoccupied molecular orbital) and their absolute energies are provided in Fig. S2 and Table S1, $\dagger$ respectively.

\section{Experimental section}

\section{Materials and reagents}

All reactions were performed in anhydrous solvents under a nitrogen atmosphere using well-dried glassware in an oven at $90{ }^{\circ} \mathrm{C}$ before use. All of the solvents, i.e., dichloromethane, chloroform, and methanol, were dried and distilled using $4 \AA$ molecular sieves. Column chromatography was performed using alumina (spherical, neutral, 63-200 $\mu \mathrm{m}$, Kishida Chemicals Co., Ltd.). HOPG was purchased from Alliance Biosystems, Inc. (Spi-1 Grade $7 \times 7 \times 1 \mathrm{~mm}$ ). The other chemicals and solvents were of reagent grade and were used without further purification. The HiPCO-SWNT samples were purchased from Carbon Nanotechnologies Inc. (diameter 0.8-1.2 $\mathrm{nm}$ and length around 100-1000 $\mathrm{nm}$ ).

\section{Synthesis of target molecules}

The target complexes, i.e., the protonated $\mathbf{1}$, anionic $\mathbf{2}$, and radical 3 forms (Scheme 1) were synthesized as depicted in Scheme S1.†

\section{Synthesis of $\left[\mathrm{Tb}^{\mathrm{III}}(\mathrm{DBPH})(\mathrm{DBP})\right]$ protonated form complex 1}

The protonated form 1 was synthesized using a previously reported method, ${ }^{46}$ where $\mathrm{Tb}^{\mathrm{III}}(\mathrm{acac})_{3} \cdot 3 \mathrm{H}_{2} \mathrm{O}(0.102 \mathrm{~g}, 0.224 \mathrm{mmol})$ and BDP (C12P) (0.102 g, $0.15 \mathrm{mmol})$ were mixed with diazabicycloundecene (DBU) $(230 \mu \mathrm{l})$ under $\mathrm{N}_{2}$ in dark conditions at $300{ }^{\circ} \mathrm{C}$ for $1.5 \mathrm{~h}$ using a Kugelrohr glass oven. The crude solid was then purified through an alumina column $\left(\mathrm{CH}_{2} \mathrm{Cl}_{2}\right)$, producing a red-brown solid. A pure crystalline powder was

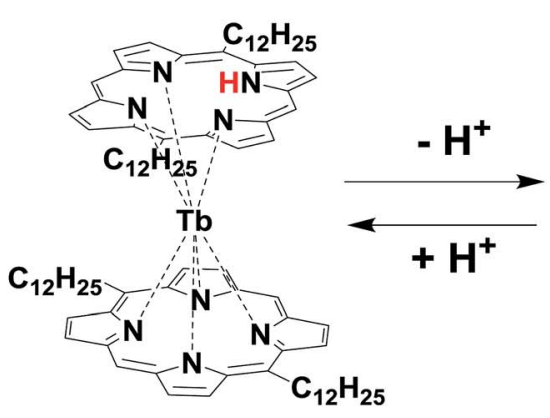

[Tb"'(BDPH)(BDP)]

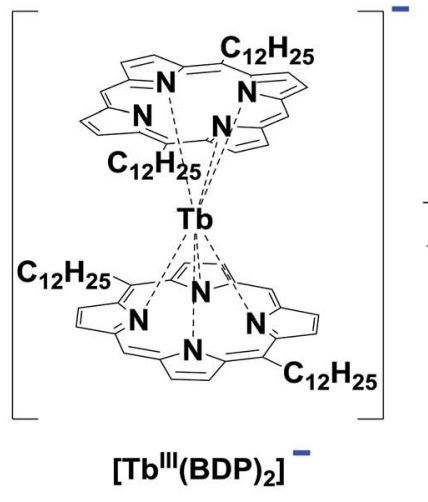

Anionic Form Complex 2

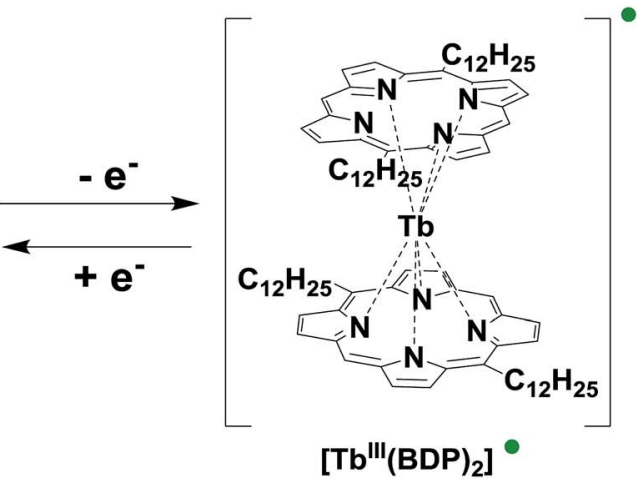

Radical Form Complex 3

Scheme 1 Interconversion between protonated form 1, anionic form 2, and radical form 3 complexes of Tb ${ }^{\text {III- }-5,15-b i s d o d e c y l p o r p h y r i n . ~}$ 
obtained by recrystallization from $\mathrm{CH}_{2} \mathrm{Cl}_{2}$ /acetonitrile $\left(\mathrm{CH}_{3} \mathrm{CN}\right)$ (42 mg, 37\%). MS (MALDI-TOF): $\mathrm{m} / z$ calcd. for $\mathrm{C}_{88} \mathrm{H}_{121} \mathrm{~N}_{8} \mathrm{~Tb}$ : $1448.9\left[\mathrm{M}^{+}\right]$; found: 1448.6.

\section{Synthesis of $\left[\mathrm{Tb}^{\mathrm{III}}(\mathrm{BDP})_{2}\right]^{-}(\mathrm{DBUH})^{+}$anionic form complex 2}

After dissolving the protonated form $\mathbf{1}(36 \mathrm{mg}, 0.025 \mathrm{mmol})$ in $\mathrm{CH}_{2} \mathrm{Cl}_{2}(10 \mathrm{ml})$, the solution was mixed with DBU $(1 \mathrm{ml})$. Then, the mixture was stirred at room temperature for 30 min in dark conditions. After evaporating the solvent, the residue was recrystallized with $\mathrm{CHCl}_{3} / \mathrm{CH}_{3} \mathrm{CN}$. The pure anionic spices were thereafter collected by filtration (34 mg, 95\%). MS (MALDITOF): $m / z$ calcd. for $\mathrm{C}_{88} \mathrm{H}_{120} \mathrm{~N}_{8} \mathrm{~Tb}: 1447.9$ [M ${ }^{+}$; found: 1447.5 .

\section{Synthesis of $\left[\mathrm{Tb}^{\mathrm{III}}(\mathrm{BDP})_{2}\right]^{\cdot}$ radical form complex 3}

A solution of the anionic form $2(46.8 \mathrm{mg}, 0.032 \mathrm{mmol})$ and $\mathrm{Fc}^{+} \mathrm{PF}_{6}{ }^{-}(5.235 \mathrm{mg}, 0.0162 \mathrm{mmol})$ in $\mathrm{CH}_{2} \mathrm{Cl}_{2}(10 \mathrm{ml})$ was stirred under $\mathrm{N}_{2}$ at room temperature for $30 \mathrm{~min}$ in dark conditions. Thereafter, the solution was concentrated and recrystallized using $\mathrm{CHCl}_{3} / \mathrm{CH}_{3} \mathrm{CN}$. The crude solid of the radical species was accumulated by filtration ( $45 \mathrm{mg}, 96 \%$ ). MS (MALDI-TOF): $\mathrm{m} / \mathrm{z}$ calcd. for $\mathrm{C}_{88} \mathrm{H}_{120} \mathrm{~N}_{8} \mathrm{~Tb}$ : 1447.9 [M ${ }^{+}$; found: 1447.9; elemental analysis calcd. (\%) for $\left(\mathrm{C}_{88} \mathrm{H}_{120} \mathrm{~N}_{8} \mathrm{~Tb}\right)(1447.9)$ : $\mathrm{C} 72.95, \mathrm{H} 8.35, \mathrm{~N}$ 7.73; found: C $72.26, \mathrm{H} 8.35$, N 7.89.

\section{Fabrication of $\mathrm{Tb}^{\mathrm{III}}$-porphyrin double-decker complex-SWNT composites}

A solution of $\mathrm{Tb}^{\mathrm{III}}$-porphyrin double-decker complex (around $225 \mu \mathrm{M})$ in dichloromethane $(20 \mathrm{ml})$ was sonicated with purified-HiPCO SWNT (15 mg) for $2 \mathrm{~h}$. The resulting suspension was then allowed to sit for $2 \mathrm{~h}$ for sedimentation. Thereafter, the top $5 \%$ of the supernatant was removed and the precipitate was filtered using a membrane filter (MILLIPORE) with a $0.1 \mu \mathrm{m}$ mesh, followed by rinsing with $100 \mathrm{ml} \mathrm{CH}_{2} \mathrm{Cl}_{2}$ to remove the non-adsorbed porphyrins. The composite was then dried in a vacuum desiccator until further utilization. For the STM measurements, the powder was re-suspended in $\mathrm{MeOH}(10 \mathrm{ml})$ and drop-casted onto the HOPG substrate.

\section{Results and discussion}

\section{UV-vis spectroscopic measurements of $\mathrm{Tb}^{\mathrm{III}}$-porphyrin BDP complexes and BDP-SWNT composites}

The BDP-SWNT composites were fabricated as described in the experimental section by sonicating the purified-HiPCO SWNT with the porphyrin solution for a while followed by sedimentation, filtration, washing and drying the resultant composites till further characterization.

The Soret peaks of the protonated form $1(392 \mathrm{~nm})$ and anionic form $2(396 \mathrm{~nm})$ were blue-shifted relative to that of the BDP (C12P) ligand (404 nm); however, this peak was red-shifted for the anionic form 2 relative to the protonated form 1 (Fig. S3 $†$ ). According to the literature, ${ }^{66-68}$ the radical forms of porphyrin and phthalocyanine double-decker complexes have a characteristic broad Q-band in the near-infrared (NIR) region together with the Soret band (UV-vis region). Therefore, an NIR absorption band was observed at $1153 \mathrm{~nm}$, in addition to the blue shift of the Soret peak of the radical form $3(387 \mathrm{~nm})$ relative to those of the BDP ligand $(404 \mathrm{~nm})$, protonated form 1 (392 nm), and anionic form $2(396 \mathrm{~nm})$. In the radical form 3, the cofacial distance between the two porphyrin rings decreases because of an increase in the bond order, due to the removal of an electron from the antibonding orbital of the HOMO, ${ }^{69-73}$ ultimately resulting in blue-shifting of the Soret band of 3 (Fig. S3†).

To confirm the interaction between the BDP molecules and SWNT surfaces, we conducted UV-vis spectroscopic measurements. Fig. 1 presents the UV-vis spectra of the pristine SWNT, protonated form 1, anionic form 2, radical form 3, 1-SWNT, 2SWNT and 3-SWNT composites in $\mathrm{CH}_{2} \mathrm{Cl}_{2}$, together with their $\lambda_{\max }$ values. The porphyrin Soret peaks are obviously Dopplershifted (to either longer or shorter wavelengths) after complexation with the SWNTs. Additionally, the composite spectra include the peaks of both porphyrin and SWNT, as can clearly be seen in Fig. 1 . For the 2 -SWNT composite $(396 \rightarrow 388$ $\mathrm{nm}$ ) the absorption of the Soret band is blue-shifted, while that of the 1-SWNT (392 $\rightarrow 304 \mathrm{~nm})$ and 3-SWNT $(387 \rightarrow 391 \mathrm{~nm})$ composites are red-shifted. These results demonstrate that the SWNTs were successfully functionalized by the porphyrin molecules. It is possibly that some charge-transfer may be occurred between the SWNT and $\mathrm{Tb}^{\mathrm{III}}$-BDP complexes as it is reported elsewhere. ${ }^{59}$

For future efficacious development of spintronic devices, one significant aspect is the fabrication of thin films and selfassembly ordered patterns of SMM materials, ${ }^{74}$ especially on nanocarbon surfaces, i.e., HOPG, graphene, and carbon nanotubes. SPM is one of the most effective techniques for studying their behaviors.

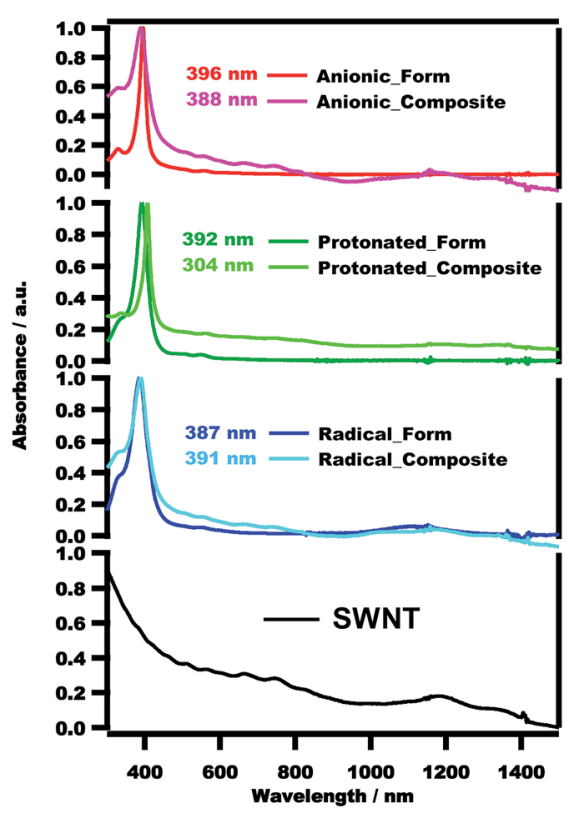

Fig. 1 UV-vis spectra of pristine SWNT, protonated form 1, anionic form 2, radical form 3, 1-SWNT, 2-SWNT and 3-SWNT composites in $\mathrm{CH}_{2} \mathrm{Cl}_{2}$, together with their $\lambda_{\max }$ values, clearly showing the Doppler shift after complexation with the SWNTs. 


\section{Ultra-high vacuum scanning tunneling microscopy at low temperatures}

The BDP-SWNT composite samples were prepared with supramolecular structures on the HOPG surface using a simple drop casting technique (i.e., drops of the dispersed composites in $\mathrm{MeOH}(c a .5 \mu \mathrm{l})$ were cast onto freshly cleaved HOPG surfaces) and fixed onto the cell. The samples were then left to dry at room temperature in preparation for observation. All images were captured under an ultra-high vacuum and low temperature $(80 \mathrm{~K})$. To induce a tunneling current, a bias voltage was applied between each sample and the tip. Image calibration was performed using Gwyddion software.

Fig. 2 shows representative STM images of the supramolecular structure of the 3-SWNT composite on the HOPG surface (additional STM images are provided in Fig. S4 $\dagger$ ). The STM images clearly exhibit two different arrangements for the $\mathbf{3}$ molecules on the SWNT surface. The first arrangement (Fig. 2a$\mathrm{d}, \mathrm{S} 4 \mathrm{a}-\mathrm{c}$ and $\mathrm{f}_{\dagger}^{\dagger}$ ) shows a regular helical supramolecular structure on the tube surface with significant debundling of the SWNT due to interaction with the adsorbed 3 . This finding suggests that the $\mathbf{3}$ molecules formed a well-ordered helixshaped array with regular periodicity on the SWNT surface stabilized by non-covalent ( $\pi$-stacking) interaction within the neighboring molecules, leading to a highly dispersed SWNT with relatively monolayer formation of the adsorbed 3 molecules on the sidewall of the nanotube. Consequently, the STM imaging results indicate that the strong interaction between the
3 molecules and SWNT were expressed not only through the debundling effect, but also through the relatively monolayer formation, which reveals the periodicity of the neighboring porphyrin molecules along the tube axis. Meanwhile, in the other arrangement, the adsorbed porphyrin forms a regular supramolecular array on the SWNT surface while interacting (both molecules and tubes) with each other side by side (Fig. 2e$\mathrm{g}, \mathrm{s} 4 \mathrm{~d}$ and $\mathrm{e} \dagger$ ).

There is a periodic structure appears on the right-hand side of the SWNT in Fig. 2a and d. Since we have used HOPG as a substrate, therefore this periodic structure due to the formation of moiré patterns form on HOPG surface. There are many reports which have discussed moiré pattern structure, ${ }^{75-77}$ which originates due to the weak van der Waals bonding between the layers of HOPG, hence, topmost layer may be shifted or rotated by mechanical or chemical means. As a result of the rotation of the top layer, super-periodic structures called as moiré patterns form on HOPG surfaces.

By using a high grade HOPG substrate, no structures have been observed during STM measuments. As by applying bias voltage to SWNT sample, the SWNT always jump and hence can't be easily detected. Therefore, we have used a lower grade of HOPG substrate where SWNT prefers to assemble near to the HOPG surface defects i.e. boundary phases. Consequently, we could successfully observe the structures of BDP/SWNT composites especially near to the boundary phases on the HOPG surface. This is can be obviously depicted in Fig. 2b, c and e. Since there is no apparent height a

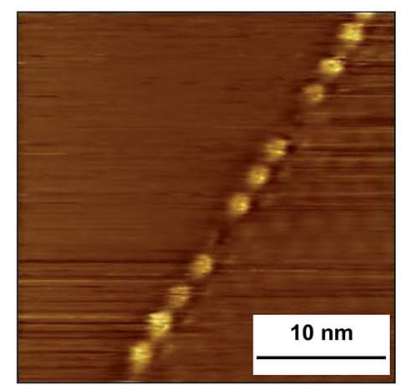

e

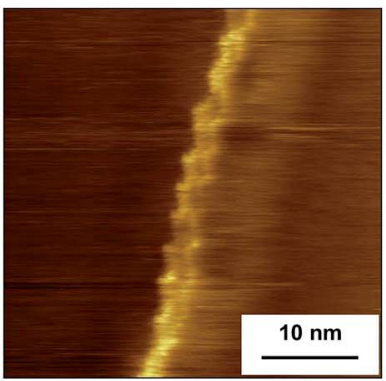

b

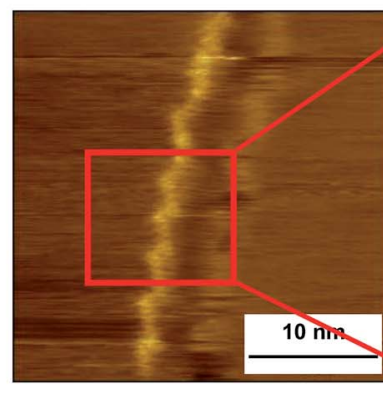

f

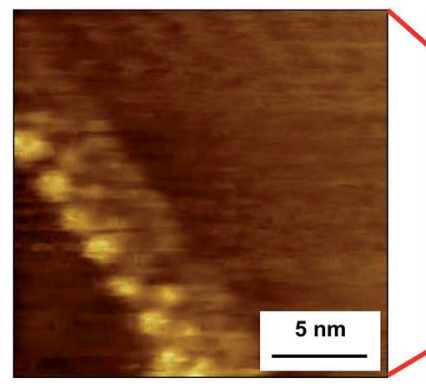

C

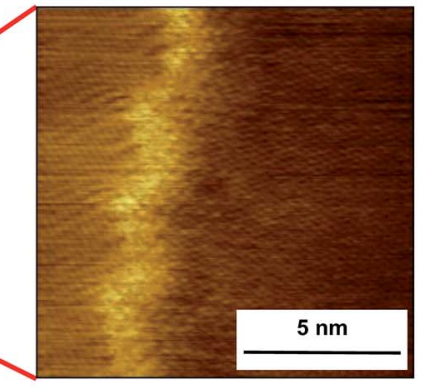

g

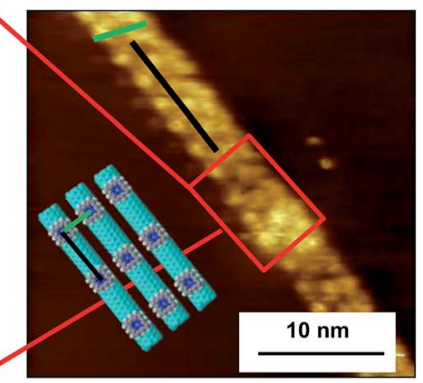

d

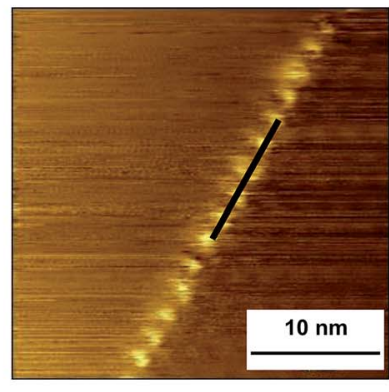

h

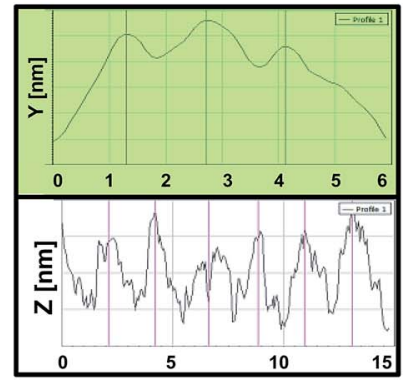

Fig. 2 Typical STM images of supramolecular structures of 3-SWNT composites on HOPG surfaces. (a) $3-S W N T$ composite $\left(I_{\mathrm{t}}=0.1 \mathrm{nA}, V_{\text {sample }}=\right.$ $0.5 \mathrm{~V}$ ). (b) 3-SWNT composite $\left(I_{\mathrm{t}}=0.1 \mathrm{nA}, V_{\text {sample }}=0.3 \mathrm{~V}\right)$. (c) 3 -SWNT composite $\left(I_{\mathrm{t}}=0.1 \mathrm{nA}, V_{\text {sample }}=0.3 \mathrm{~V}\right)$. (d) 3 -SWNT composite $\left(I_{\mathrm{t}}=0.05\right.$ $\left.\mathrm{nA}, V_{\text {sample }}=0.2 \mathrm{~V}\right)$. (e) 3-SWNT composite $\left(I_{\mathrm{t}}=0.1 \mathrm{nA}, V_{\text {sample }}=0.1 \mathrm{~V}\right)$. (f) 3 -SWNT composite $\left(I_{\mathrm{t}}=0.05 \mathrm{nA}, V_{\text {sample }}=0.1 \mathrm{~V}\right)$. (g) $3-\mathrm{SWNT}$ composite $\left(I_{\mathrm{t}}=0.05 \mathrm{nA}, V_{\text {sample }}=-0.08 \mathrm{~V}\right)$ with schematic model drawn to elucidate the side by side interactions of the composites. (h) Topographic profiles of 3-SWNT composite, where the upper part represents the observed average pitch distance (average distance of periodicity of the helix), which is $2.3 \pm 0.1 \mathrm{~nm}$ (marked with the green line in g), while the lower part shows that the center-to-center distance between two adjacent SWNTs is $1.4 \mathrm{~nm}$ (marked with the black lines in $\mathrm{d}$ and $\mathrm{g}$ ). 
difference in the STM images around the BDP-tube composites, the composite structures can be clearly distinguished from HOPG step-terrace structures.

Since the used raw-HiPCO SWNT sample includes a mixture of chiral and achiral SWNTs i.e. $(9,6),(9,7),(8,7),(7,7),(12,3)$, $(13,4),(8,8),(10,0),(11,0)$ with different $(m, n)$ as well as handedness chiralities, as it is clearly depicted in Fig. S1. $\dagger$ Therefore, different wrapping patterns have been observed where Tb-BDP molecules form different helical structures on the tube surface, which are in quite agreement with those results of nonextracted raw-SWNTs ${ }^{78-80}$ and quite different from those results of extracted chiral-SWNT. ${ }^{65}$ This is can be obviously seen in Fig. 2 and S4. $\dagger$

The observed average pitch distance (average distance of periodicity of the helix) is $2.3 \pm 0.1 \mathrm{~nm}$, which is smaller than that we obtained previously with a BDP ligand on an SWNT surface $^{65}$ (i.e., $\left.4 \pm 0.5 \mathrm{~nm}\right),{ }^{65}$ possibly because stronger closepacking arises from the presence of radical 3 molecules. Additionally, the center-to-center distance between two adjacent SWNTs is $1.4 \mathrm{~nm}$, as depicted in the topographic profile in Fig. 2h. Accordingly, the short center-to-center distance also suggests the formation of a short helix-shaped array, which is in agreement with the results reported elsewhere. ${ }^{78,79}$

\section{AFM under ambient conditions}

AFM was used as an effective tool to investigate the supramolecular structure of 3 on the SWNT surface, by casting a few drops of the dispersed composite in $\mathrm{MeOH}$ onto a freshly cleaved HOPG surface. Fig. 3 displays typical AFM images (topographic and phase) of the composite under ambient conditions (additional AFM images are provided in Fig. S5 $\dagger$ ). As in the STM images, a distinctive debundling effect is clearly observable due to the molecular adsorption on the SWNT surface (Fig. 3a-d) with some helical array structures, as depicted in Fig. $3 \mathrm{a}$ and $\mathrm{d}$ and represented in the schematic model in Fig. 3h. Additionally, interacting composites (bundled together) are observable in Fig. $3 \mathrm{e}-\mathrm{g}$, in agreement with our STM observations (Fig. 2e-g). The average height of the 3-SWNT composite is approximately $0.677 \mathrm{~nm}$, as shown in the topographic profiles in Fig. S5d and $\mathrm{S} 5 \mathrm{~h}, \dagger$ which is quite reasonable compared to the reported one with pyrenyl moieties. ${ }^{59}$

\section{Scanning electron microscopy (SEM) coupled energy dispersive X-ray spectroscopy (EDS) measurements}

We performed SEM-coupled EDS analysis to characterize the 3SWNT composite qualitatively and quantitatively. Fig. 4 presents the SEM-coupled EDS images together with the spatial distribution of metal ions in the sample, in which one can recognize obviously the presence of $\mathrm{Tb}$ ions as evidence that the SWNT was successfully functionalized with the molecule. Quantitatively, we estimated the molecular content in the BDP-SWNT hybrid using EDS, where the weight content of Tb was first estimated to be approximately $0.14 \%$ by weight. Thereafter, the BDP-Tb double-decker complex content was estimated by recalculating the value for the total molecular weight of the BDP-Tb complex based on the atomic weight of $\mathrm{Tb}$ to be about $1.28 \%$. Although the SWNT surface was not fully covered by BDP-Tb molecules (due to the low molecular concentration on the tube surface), this result may suggest the formation of a relatively uniform monolayer of the adsorbed a

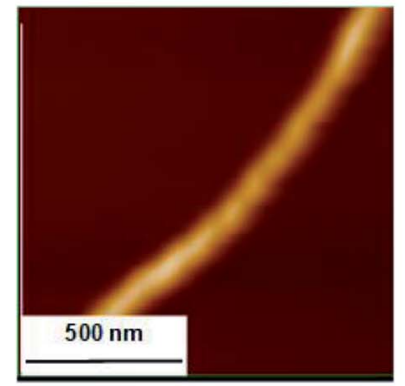

e

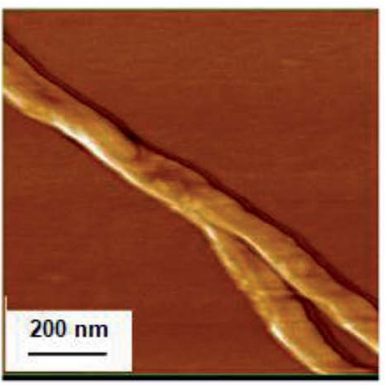

b

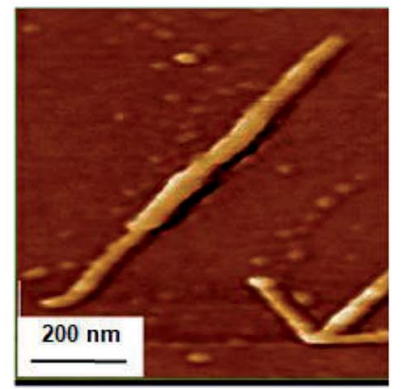

$\mathbf{f}$

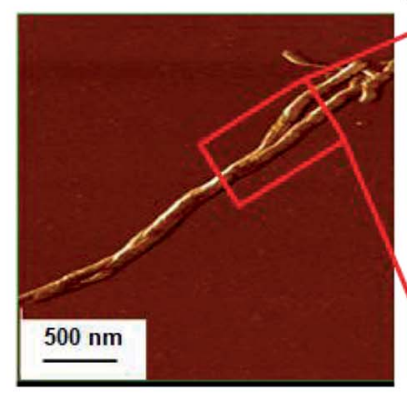

C

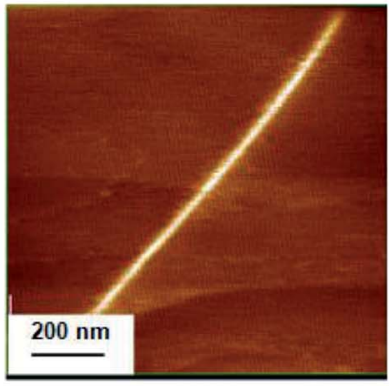

g

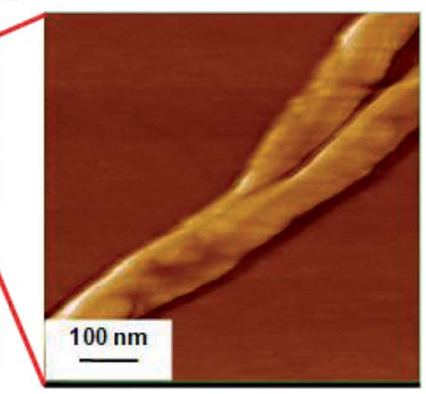

d
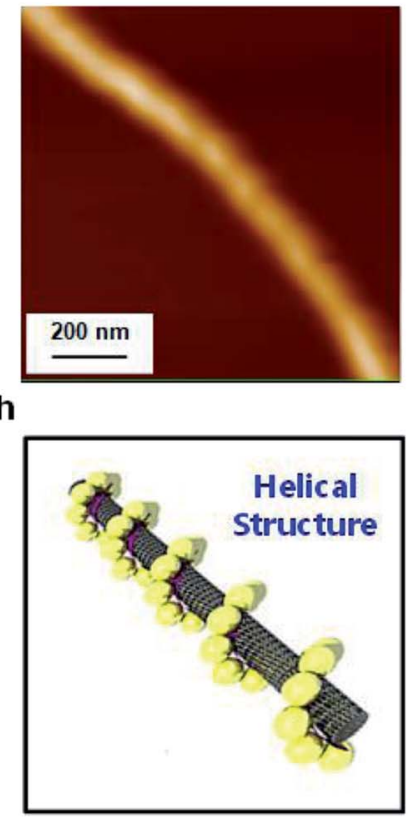

Fig. 3 (a-g) Typical AFM images (topographic and phase) of supramolecular structures of 3-SWNT composites on HOPG surfaces under ambient conditions. (h) Schematic model representing the helical structure formed by a radical form 3 molecule on the SWNT surface, as in a and d. 
a

b
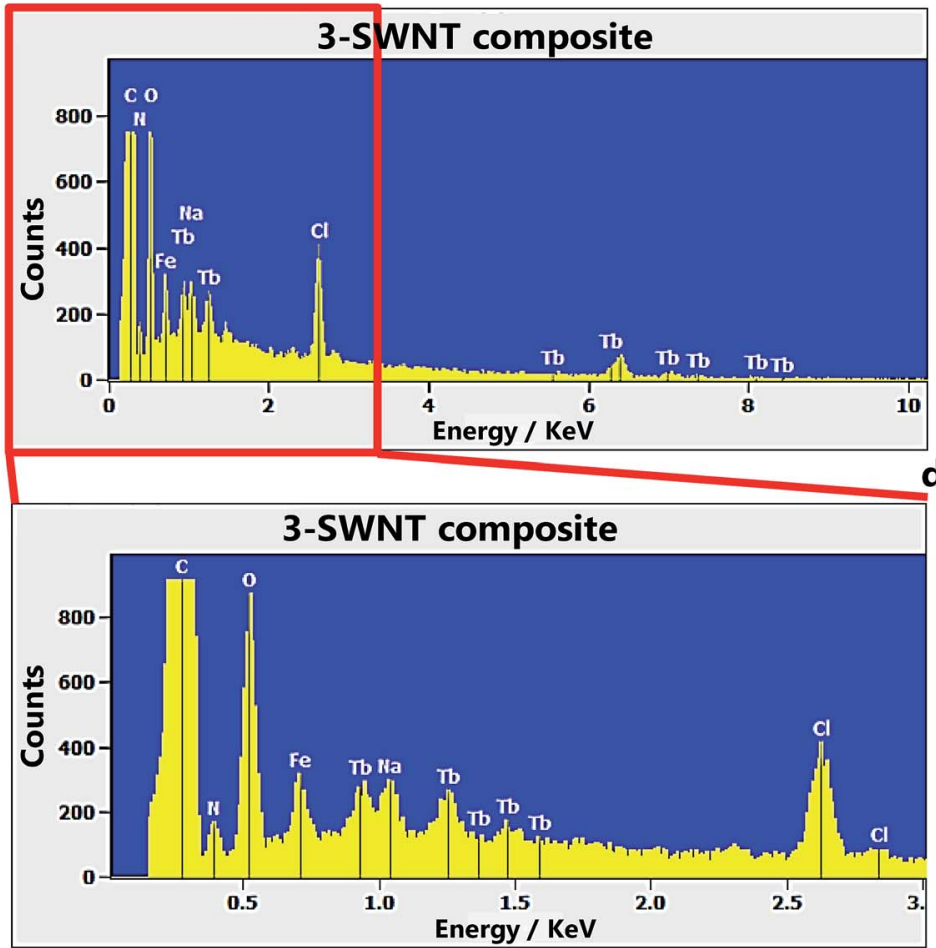

C
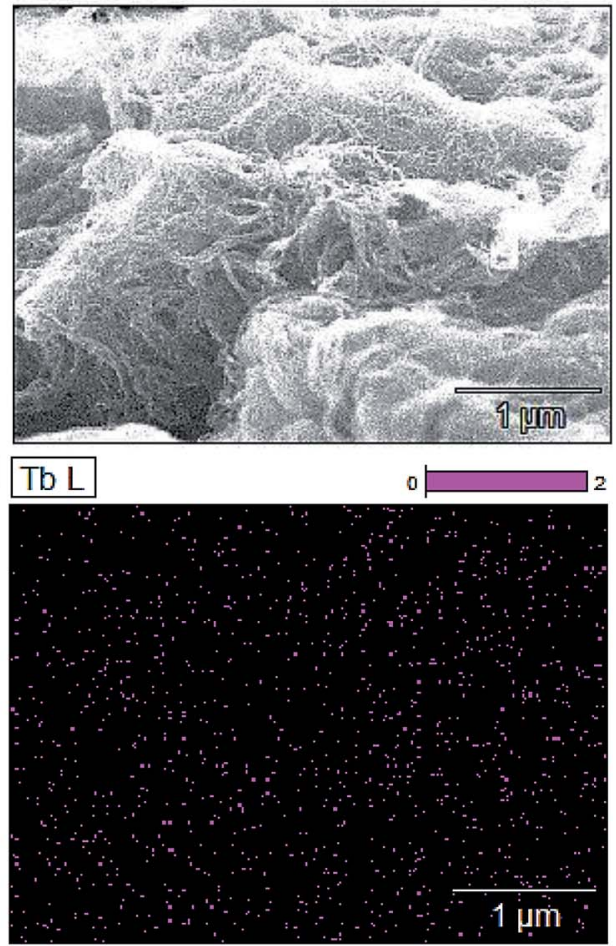

Fig. 4 ( $a$ and b) The EDX spectra of the 3-SWNT composite. (c), SEM image of 3-SWNT composite. (d) The spatial distributions of Tb ions in the samples.

molecules on the SWNT surface, as confirmed by the STM and AFM results.

\section{Magnetic measurements}

To investigate the magnetic properties of all the BDP-Tb doubledecker complexes, we performed ac magnetic susceptibility measurements for protonated form 1, anionic form 2, and radical form 3 complexes under both 0 and 2000 Oe dc magnetic fields, as depicted in Fig. 5a-d and S6. $\dagger$ Fig. S6e and $\mathrm{f} \dagger$ demonstrate that the protonated form complex $\mathbf{1}$ does not act as an SMM, possibly due to the low molecular symmetry. This finding agrees with our previous research, ${ }^{45,46,48}$ in which the protonated forms of some $\mathrm{Tb}^{\mathrm{III}}$-porphyrin double-decker complexes did not exhibit SMM properties due to their low symmetric coordination environments. In contrast, the anionic form 2 and radical form 3 complexes exhibited clear slow magnetic relaxation, as can be seen in Fig. $5 \mathrm{a}-\mathrm{d}$ and $\mathrm{S} 6 \mathrm{a}-\mathrm{d}, \uparrow$ which represent the temperature dependence of the in-phase $\left(\chi_{M}^{\prime}\right)$ and out-of-phase $\left(\chi_{M}^{\prime \prime}\right)$ ac magnetic susceptibilities of the powder samples under 0 and 2000 Oe dc magnetic fields. Complexes 2 and 3 both act as SMMs at the blocking temperature of $27 \mathrm{~K}$ (shown by the $\chi_{\mathrm{M}}^{\prime}$ and $\chi_{\mathrm{M}}^{\prime \prime}$ peak temperatures under an ac field of $1000 \mathrm{~Hz}$ ), which is slightly higher than the reported temperature for $\mathrm{Tb}^{\mathrm{III}}$-porphyrin double-decker complexes (24 K). ${ }^{\mathbf{4 6}, 48}$ By measuring the dc magnetizations of complexes 2 and 3 to investigate the magnetic field $(H)$ dependence of the magnetization $(M)$ at low temperature, i.e., $1.8 \mathrm{~K}$ within \pm 20 kOe, butterfly-shaped hysteresis loops were observed, as displayed in Fig. 5e and f.

After mixing with SWNT, the magnetic behaviors of both the 2-SWNT and 3-SWNT composites were examined through their ac susceptibilities and dc magnetizations using a superconducting quantum interference device magnetometer. However, the ac susceptibility measurements did not allow accurate comparison with the powder crystalline samples of compounds 2 and 3 due to the broadening of the peaks as well as the low signals. Consequently, no clear ac susceptibility peaks are observable for the blocking temperature (Fig. S7 and S8†), possibly due to the low molecular concentration on the tube surface, which may suppress the molecule-tube interactions, and hence no obvious blocking temperature peaks are recognizable in the ac susceptibility measurements.

On the other hand, we measured the dc magnetizations for both the 2-SWNT and 3-SWNT composites. Fig. 6a-d depict the temperature dependence (from 2 to $300 \mathrm{~K}$ ) of the dc magnetic susceptibility measurements at a constant magnetic field, i.e., 2 kOe for the 2-SWNT and 3-SWNT composites, where the molar magnetic susceptibility-temperature product $(\chi T)$ increases almost linearly with increasing temperature within the measurement range. However this behavior is similar to the reported one for $\mathrm{Tb}$-octa(ethyl)tetraazaporphyrin ${ }^{81}$ but not exactly same where the presence of SWNT may give some potential to the SMM character.

To investigate the $H$ dependence of $M$ at low temperatures, where distinctive opened hysteresis cycles were observed. 
a

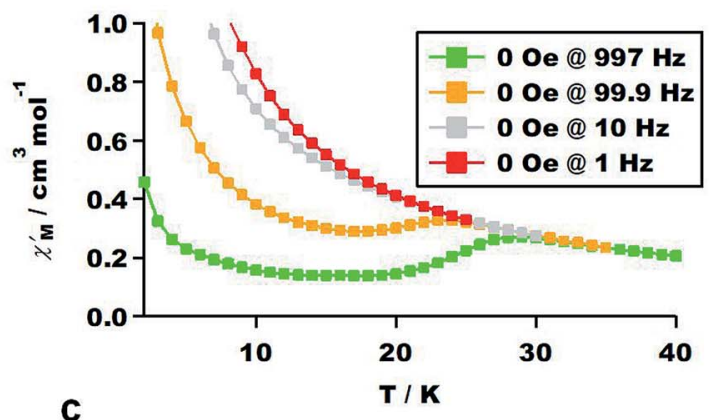

C
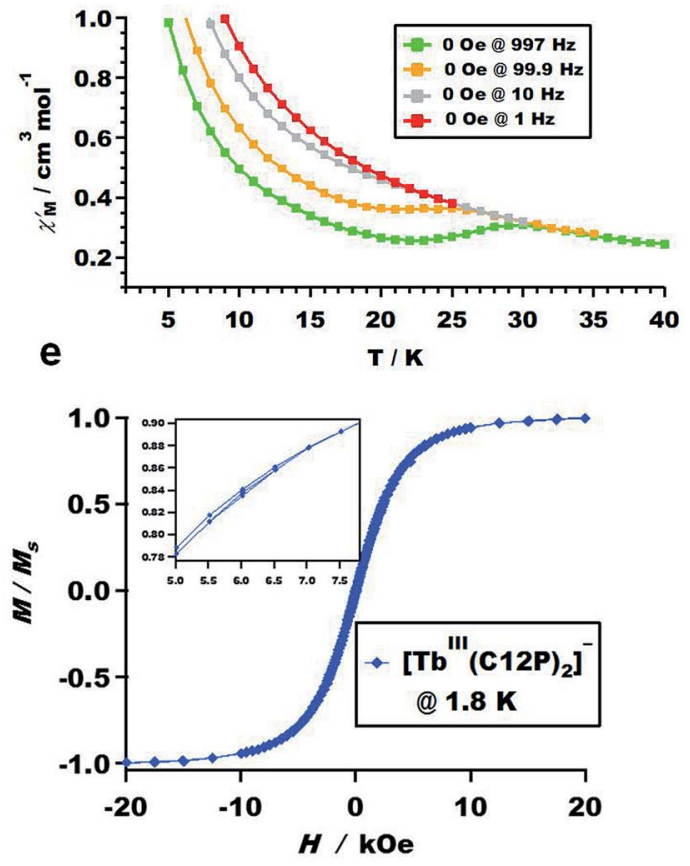

b
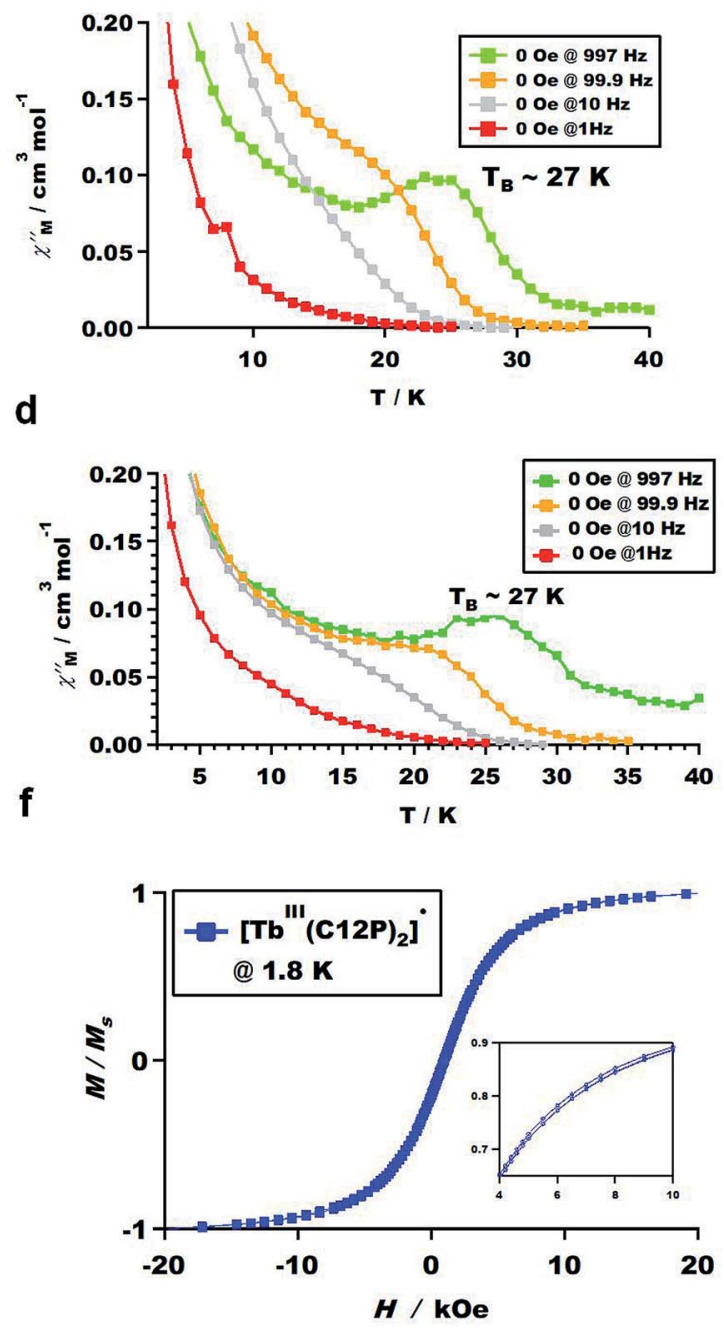

Fig. 5 Temperature dependences of the in-phase $\left(\chi_{M}^{\prime}\right)$ and out-of-phase $\left(\chi_{M}^{\prime \prime}\right)$ ac magnetic susceptibilities of (a and b) the anionic form 2 and (c and d) the radical form 3 under 0 Oe dc magnetic field. Hysteresis loops of (e) the anionic form 2 and (f) the radical form 3 at $1.8 \mathrm{~K}$ within $\pm 20 \mathrm{kOe}$.

Fig. 6e and $\mathrm{f}$ display the butterfly-shaped hysteresis loops of the 2-SWNT and 3-SWNT composites at $1.8 \mathrm{~K}$ within $\pm 20 \mathrm{kOe}$. The butterfly-shaped loops are notably more open for both the 2SWNT and 3-SWNT composites compared to those of the molecules alone (Fig. 5e and f). These results hint at longer relaxation times for the composites in the magnetic field region in which the butterfly-shaped loops are open. This finding is in agreement with other literature,${ }^{59}$ which states that the presence of HiPCO SWNTs increases the relaxation time of the SMMSWNT conjugates. In contrast, other reports state that the relaxation time decreases for $\mathrm{Fe}_{6}$-based SMMs ${ }^{61}$ and $\mathrm{Mn}_{12}$-based $\mathrm{SMMS}^{82}$ after complexing with CNTs, which is possibly due to the higher sensitivity to small structural deformations of polynuclear transition-metal SMMs compared to that of monocenter rare-earth SMMs. ${ }^{59}$

To show the contribution of magnetic adsorbates ( 2 and 3 ) on SWNTs, we have measured the hysteresis loop of the 1-SWNT (Fig. S9†). Interestingly, smaller hysteresis loop has been observed for 1-SWNT compared to 2-SWNT and 3-SWNT composites. This suggests that the presence of protonated form 1 (SMM OFF) suppresses the magnetic properties of the SWNT as well as the resultant composite 1-SWNT. In contrast, larger hysteresis loops have been observed for 2-SWNT and 3-SWNT composites which possibly due to the coupling between adsorbates anionic form 2 and radical form 3 molecules (SMMs ON) with the paramagnetic SWNTs without neglecting the iron residues, displaying the contribution of magnetic adsorbates $(2$ and 3) on SWNTs.

To explain why no clear ac susceptibility peaks are evident for the 2-SWNT and 3-SWNT composites however an open hysteresis behavior like clear SMM system was observed by measuring the dc magnetizations, as well as what the SWNT potential to the SMM character, we measured the temperature dependence of the dc magnetic susceptibility for the purifiedHiPCO SWNT sample only at a constant magnetic field of 2 kOe. As is obvious in Fig. S10, $\uparrow$ the presence of a large content of 


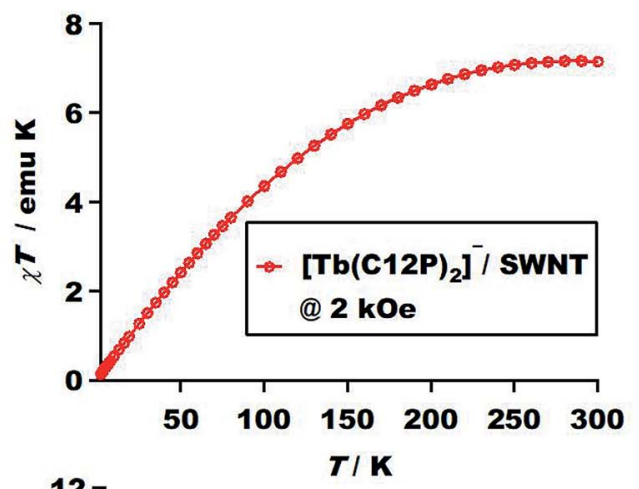

C
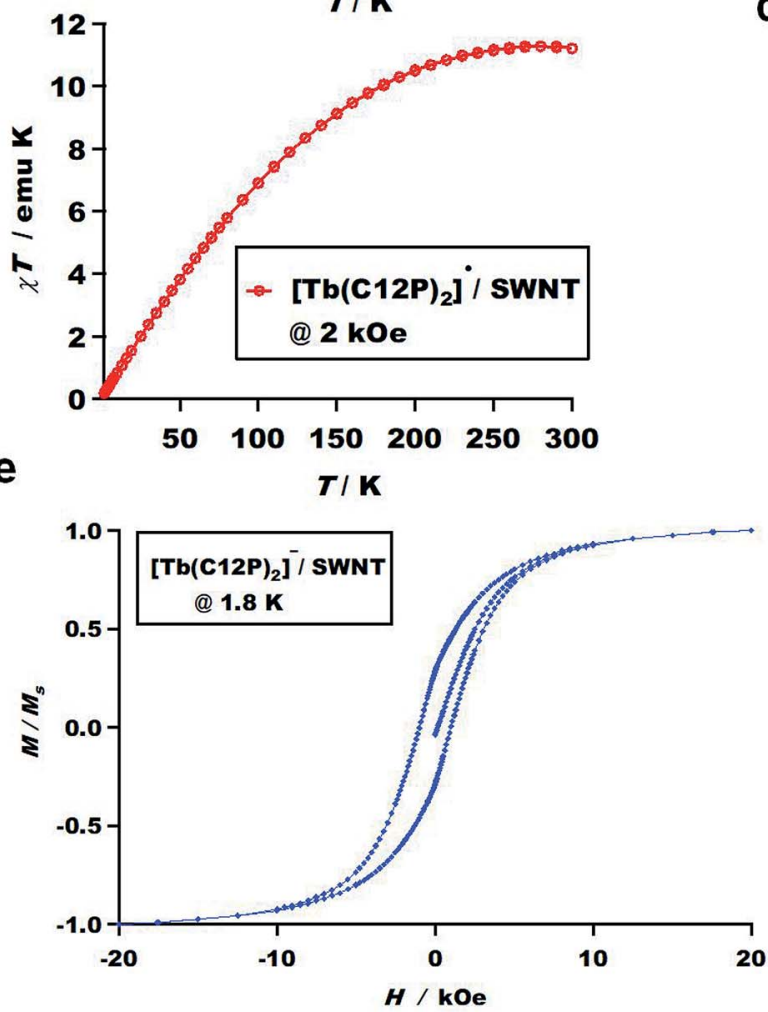

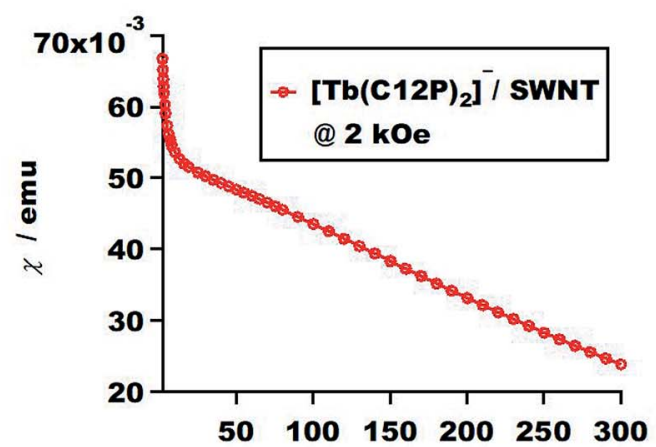

d

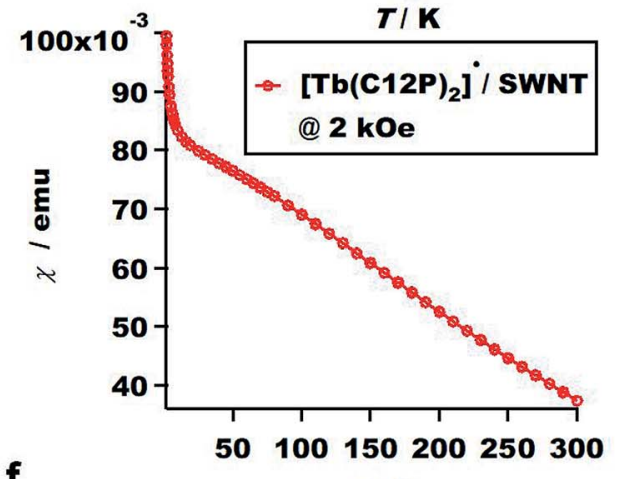

$\mathbf{f}$

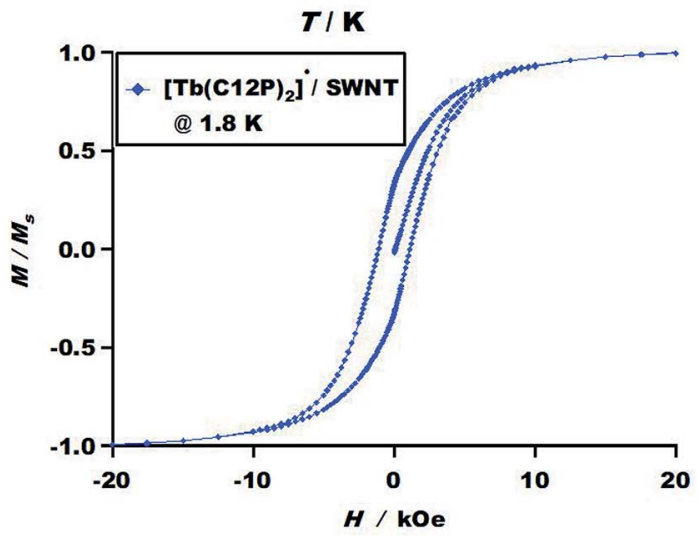

Fig. 6 Temperature dependence of the dc magnetic susceptibility measurements of ( $a$ and b) the 2-SWNT composite, (c and d) the 3-SWNT composite. Hysteresis loops of (e) the 2-SWNT composite and (f) the 3-SWNT composite at $1.8 \mathrm{~K}$ within $\pm 20 \mathrm{kOe}$.

SWNTs (compared to the low molecular concentration) enhances the anti-ferromagnetic coupling between the SWNT and porphyrin molecules and subsequently suppresses the molecule-tube interactions. Hence, there are no obvious blocking temperature peaks in the ac susceptibility measurements.

The magnetic properties of carbon nanotubes (CNTs) are reported $^{\mathbf{8 3 - 8 5}}$ due to their sensitivity to the applied magnetic field especially if the magnetic field direction is parallel to the tube principle axis, therefore CNTs may show some response to the applied magnetic field (paramagnetic or diamagnetic) depending on some parameters i.e. tube's diameter, chirality, Fermi energy level, and the magnetic field direction relative to tube principle axis. Consequently, by performing SQUID measurements for purified-HiPCO SWNT, however no clear identification has been concluded from the ac susceptibility measurements (Fig. S11a-d†) but interestingly a notable large hysteresis loop was observed (Fig. S11g $\dagger$ ). Additionally, SEM coupled EDS was measured and reveals the presence of some iron residues as shown in Fig. S12. $\dagger$ Basically, we have tried to completely purify the SWNT sample from the catalyst presence, but it is a matter of difficulty to remove the metal catalysts completely from the raw-SWNT sample.

The origin of SWNT magnetization (whether it is from SWNT sensitivity to the applied field or due to the presence of iron residues) was revealed by measuring the temperature dependence of the magnetic susceptibility of purified-HiPCO SWNT at different applied magnetic field between 50 Oe and $4 \mathrm{~T}$ for temperatures below $100 \mathrm{~K}$, and up to $7 \mathrm{~T}$ at $300 \mathrm{~K}$ (Fig. S11e and $\mathrm{f}^{\dagger}$ ). The figure displays that the magnetic susceptibility $\chi$ steadily decreases by 
increasing the applied magnetic field and the temperature increase which indicates the presence of predominant antiferromagnetic interactions, that's in agreement with the reported results. ${ }^{86}$ Thereof, in addition to the presence of iron catalyst particles, there is also possibility that SWNT shows magnetic response to the applied magnetic field which seems to be happened in our SWNT sample and resulted in the remarkable opened hysteresis loop in Fig. S11g. $\dagger$ Moreover, since the magnetic saturation value of the iron spin system equals $100-150 \mathrm{emu} \mathrm{g}^{-1}$ within $\pm 10 \mathrm{kOe}^{87}$ which in comparison with that of our purifiedHiPCO SWNT equals 8-10 emu $\mathrm{g}^{-1}$, this is also other evidence that the SWNT magnetization origin is not only from the iron particles but also possibly from the SWNT itself.

This is possibly explain why the composite magnetization increases almost linearly with decreasing temperature (Fig. 6ad) as well as the large hysteresis loops (Fig. 6e and f), where the SWNT with low percentage of iron residual shows the same behavior, ${ }^{83}$ which may be possibly caused by the coupling between porphyrin molecules with the paramagnetic SWNTs without neglecting the iron residues.

\section{Conclusions}

We successfully observed the supramolecular structures of the $\mathrm{Tb}^{\mathrm{III}}$-porphyrin double-decker complex-SWNT composites on the surfaces of SWNTs by performing STM as well as AFM. STM revealed well-ordered, self-assembled, helix-shaped arrays with regular periodicity on the SWNT surface stabilized with noncovalent ( $\pi$-stacking) interactions, while the AFM images exhibited a distinctive debundling effect due to BDP-molecules adsorption on the SWNT surface with some helical array structures. The composites were characterized by UV-vis spectroscopy, which revealed Doppler shifts of the molecule spectra after complexation with the SWNTs. Additionally, the magnetic measurements of the $\mathrm{Tb}^{\mathrm{III}}$-porphyrin double-decker complexes exhibited clear slow magnetic relaxation, where the complexes acted as SMMs at the blocking temperature of $27 \mathrm{~K}$ and butterfly-shaped hysteresis loops were observed. Moreover, after mixing with the SWNTs, some magnetic properties of the BDP-SWNT composites were studied where the composites interestingly show larger butterfly-shaped hysteresis loops than the molecule alone, indicating the longer relaxation times of the composites. Moreover, the composite magnetization increases almost linearly with decreasing temperature which possibly due to the coupling between BDP molecules with the paramagnetic SWNTs without neglecting the iron residues. To the best of our knowledge, this is the first report to describe in detail the supramolecular structures of based $\mathrm{Tb}^{\mathrm{III}}$ double-decker complex on the surfaces of SWNTs using SPM imaging techniques. However, supramolecular structures on SWNT surfaces are still quite challenging to predict and understand. This study will pave the way for the future development of spintronic devices based on SMM-nanocarbon composites as well as open a path towards further understanding and the building of new supramolecular architectures on SWNT surfaces.

\section{Conflicts of interest}

The authors declare no competing financial interests.

\section{Acknowledgements}

This study was supported in part by Grants-in-Aid for Scientific Research on Innovative Areas from the Ministry of Education, Culture, Science, Sports, and Technology of Japan No. 25110002. One of the authors (Dr Ahmed I. A. Abd El-Mageed) would like to gratefully acknowledge the financial support granted by the International Joint Research Promotion Program of Osaka University, the Nanotech CUPAL NIP course (National Institute of Material Science, Tsukuba) for the financial support during the course, and Prof. Daisuke Fujita and Dr Nobuyuki Ishida (National Institute of Material Science, Tsukuba) for hosting Dr Abd El-Mageed during the STM measurements. The authors also thank Dr Satoshi Yamashita (Osaka University) and Dr Ken-ichi Yamashita (Osaka University) for the valuable discussions as well as their help in the performance of SQUID measuments. The DFT calculations were performed at the Research Center for Computational Science, Okazaki, Japan.

\section{References}

1 P. Avouris, Acc. Chem. Res., 2002, 35, 1026-1034.

2 M. Urdampilleta, S. Klyatskaya, J.-P. Cleuziou, M. Ruben and W. Wernsdorfer, Nat. Mater., 2011, 10, 502.

3 S. J. Tans, A. R. Verschueren and C. Dekker, Nature, 1998, 393, 49-52.

4 T. Zhang, M. B. Nix, B. Y. Yoo, M. A. Deshusses and N. V. Myung, Electroanalysis, 2006, 18, 1153-1158.

5 J. Kong, N. R. Franklin, C. Zhou, M. G. Chapline, S. Peng, K. Cho and H. Dai, Science, 2000, 287, 622-625.

6 J. Li, Y. Lu, Q. Ye, M. Cinke, J. Han and M. Meyyappan, Nano Lett., 2003, 3, 929-933.

7 C. Gao, Z. Guo, J.-H. Liu and X.-J. Huang, Nanoscale, 2012, 4, 1948-1963.

8 Z. Liu, S. Tabakman, K. Welsher and H. Dai, Nano Res., 2009, $2,85-120$.

9 T. Akasaka, F. Watari, Y. Sato and K. Tohji, Mater. Sci. Eng., C, 2006, 26, 675-678.

10 P. Serp, M. Corrias and P. Kalck, Appl. Catal., A, 2003, 253, 337-358.

11 J. Planeix, N. Coustel, B. Coq, V. Brotons, P. Kumbhar, R. Dutartre, P. Geneste, P. Bernier and P. Ajayan, J. Am. Chem. Soc., 1994, 116, 7935-7936.

12 M. S. Dresselhaus, G. Dresselhaus and A. Jorio, Annu. Rev. Mater. Res., 2004, 34, 247-278.

13 K. S. Fernando, Y. Lin, W. Wang, S. Kumar, B. Zhou, S. Y. Xie, L. T. Cureton and Y. P. Sun, J. Am. Chem. Soc., 2004, 126, 10234-10235.

14 Q. W. Li, J. Zhang, H. Yan, M. S. He and Z. F. Liu, Carbon, 2004, 42, 287-291.

15 J. Zhou, Y. Maeda, J. Lu, A. Tashiro, T. Hasegawa, G. F. Luo, L. Wang, L. Lai, T. Akasaka and S. Nagase, Small, 2009, 5, 244-255. 
16 N. R. Franklin, Q. Wang, T. W. Tombler, A. Javey, M. Shim and H. Dai, Appl. Phys. Lett., 2002, 81, 913-915.

17 M. S. Fuhrer, B. M. Kim, T. Durkop and T. Brintlinger, Nano Lett., 2002, 2, 755-759.

18 C. Subramaniam, T. Sreeprasad, T. Pradeep, G. Pavan Kumar, C. Narayana, T. Yajima, Y. Sugawara, H. Tanaka, T. Ogawa and J. Chakrabarti, Phys. Rev. Lett., 2007, 99, 167404.

19 X. Z. Bo, N. G. Tassi, C. Y. Lee, M. S. Strano, C. Nuckolls and G. B. Blanchet, Appl. Phys. Lett., 2005, 87, 203510.

20 T. Takenobu, T. Takano, M. Shiraishi, Y. Murakami, M. Ata, H. Kataura, Y. Achiba and Y. Iwasa, Nat. Mater., 2003, 2, 683688.

21 A. K. Feldman, M. L. Steigerwald, X. F. Guo and C. Nuckolls, Acc. Chem. Res., 2008, 41, 1731-1741.

22 P. M. Vora, O. N. Torrens and J. M. Kikkawa, Carbon, 2012, 50(3), 771-777.

23 E. Abou-Hamad, Y. Kim, M. Bouhrara, Y. Saih, T. Wågberg, D. E. Luzzi and C. Goze-Bac, Phys. B, 2012, 407(4), 740-742.

24 M. Kibalchenko, M. Payne and J. Yates, ACS Nano, 2011, 5, 537-545.

25 R. Sessoli, D. Gatteschi, A. Caneschi and M. Novak, Nature, 1993, 365, 141.

26 A. Cornia, M. Mannini, P. Sainctavit and R. Sessoli, Chem. Soc. Rev., 2011, 40, 3076-3091.

27 T. Glaser, Chem. Commun., 2011, 47, 116-130.

28 J. S. Miller and D. Gatteschi, Chem. Soc. Rev., 2011, 40, 30653066.

29 J. D. Rinehart and J. R. Long, Chem. Sci., 2011, 2, 2078-2085.

30 S. Sanvito, Chem. Soc. Rev., 2011, 40, 3336-3355.

31 L. Sorace, C. Benelli and D. Gatteschi, Chem. Soc. Rev., 2011, 40, 3092-3104.

32 N. Domingo, E. Bellido and D. Ruiz-Molina, Chem. Soc. Rev., 2012, 41, 258-302.

33 I.-R. Jeon and R. Clérac, Dalton Trans., 2012, 41, 9569-9586.

34 F. Habib and M. Murugesu, Chem. Soc. Rev., 2013, 42, 32783288.

35 N. Ishikawa, Polyhedron, 2007, 26, 2147-2153.

36 L. Bogani and W. Wernsdorfer, in Nanoscience And Technology: A Collection of Reviews from Nature Journals, World Scientific, 2010, pp. 194-201.

37 N. Ishikawa, M. Sugita, T. Ishikawa, S.-y. Koshihara and Y. Kaizu, J. Am. Chem. Soc., 2003, 125, 8694-8695.

38 N. Ishikawa, M. Sugita, N. Tanaka, T. Ishikawa, S.-y. Koshihara and Y. Kaizu, Inorg. Chem., 2004, 43, 54985500.

39 S. Takamatsu, T. Ishikawa, S.-y. Koshihara and N. Ishikawa, Inorg. Chem., 2007, 46, 7250-7252.

40 M. A. AlDamen, J. M. Clemente-Juan, E. Coronado, C. MartíGastaldo and A. Gaita-Arino, J. Am. Chem. Soc., 2008, 130, 8874-8875.

41 S.-D. Jiang, B.-W. Wang, H.-L. Sun, Z.-M. Wang and S. Gao, J. Am. Chem. Soc., 2011, 133, 4730-4733.

42 A. Yamashita, A. Watanabe, S. Akine, T. Nabeshima, M. Nakano, T. Yamamura and T. Kajiwara, Angew. Chem., Int. Ed., 2011, 50, 4016-4019.
43 J. D. Rinehart, M. Fang, W. J. Evans and J. R. Long, Nat. Chem., 2011, 3, 538.

44 D. N. Woodruff, R. E. Winpenny and R. A. Layfield, Chem. Rev., 2013, 113, 5110-5148.

45 D. Tanaka, T. Inose, S. Shimono, H. Tanaka, T. Tamaki, A. I. A. El-Mageed, A. K. Dyab, N. Ishikawa and T. Ogawa, e-J. Surf. Sci. Nanotechnol., 2014, 12, 124-128.

46 T. Inose, D. Tanaka, H. Tanaka, O. Ivasenko, T. Nagata, Y. Ohta, S. De Feyter, N. Ishikawa and T. Ogawa, Chem.Eur. J., 2014, 20, 11362-11369.

47 K. Katoh, Y. Horii, N. Yasuda, W. Wernsdorfer, K. Toriumi, B. K. Breedlove and M. Yamashita, Dalton Trans., 2012, 41, 13582-13600.

48 D. Tanaka, T. Inose, H. Tanaka, S. Lee, N. Ishikawa and T. Ogawa, Chem. Commun., 2012, 48, 7796-7798.

49 T. Fukuda, K. Matsumura and N. Ishikawa, J. Phys. Chem. A, 2013, 117, 10447-10454.

50 H. Wang, K. Wang, J. Tao and J. Jiang, Chem. Commun., 2012, 48, 2973-2975.

51 K.-i. Yamashita, N. Sakata and T. Ogawa, Inorg. Chem., 2016, 55, 8935-8942.

52 R. Guilard, K. M. Kadish, K. M. Smith and R. Guilard, The porphyrin handbook, Academic Press, New York, 2003.

53 T. Inose, D. Tanaka, O. Ivasenko, K. Tahara, S. De Feyter, Y. Tobe, H. Tanaka and T. Ogawa, Chem. Lett., 2016, 45, 286-288.

54 M. Gonidec, R. Biagi, V. Corradini, F. Moro, V. De Renzi, U. del Pennino, D. Summa, L. Muccioli, C. Zannoni and D. B. Amabilino, J. Am. Chem. Soc., 2011, 133, 6603-6612.

55 J. Otsuki, Y. Komatsu, D. Kobayashi, M. Asakawa and K. Miyake, J. Am. Chem. Soc., 2010, 132, 6870-6871.

56 E. M. Pineda, T. Komeda, K. Katoh, M. Yamashita and M. Ruben, Dalton Trans., 2016, 45, 18417-18433.

57 J. Hellerstedt, A. Cahlík, M. Švec, B. de la Torre, M. MoroLagares, T. Chutora, B. Papoušková, G. Zoppellaro, P. Mutombo and M. Ruben, Nanoscale, 2018, 10, 1555315563.

58 J. Gomez-Segura, I. Diez-Perez, N. Ishikawa, M. Nakano, J. Veciana and D. Ruiz-Molina, Chem. Commun., 2006, 2866-2868.

59 S. Kyatskaya, J. R. n. Galán Mascarós, L. Bogani, F. Hennrich, M. Kappes, W. Wernsdorfer and M. Ruben, J. Am. Chem. Soc., 2009, 131, 15143-15151.

60 L. Bogani, C. Danieli, E. Biavardi, N. Bendiab, A. L. Barra, E. Dalcanale, W. Wernsdorfer and A. Cornia, Angew. Chem., Int. Ed., 2009, 48, 746-750.

61 A. Giusti, G. Charron, S. Mazerat, J. D. Compain, P. Mialane, A. Dolbecq, E. Rivière, W. Wernsdorfer, R. Ngo Biboum and B. Keita, Angew. Chem., Int. Ed., 2009, 48, 4949-4952.

62 M. Ganzhorn, S. Klyatskaya, M. Ruben and W. Wernsdorfer, ACS Nano, 2013, 7, 6225-6236.

63 M. Urdampilleta, N.-V. Nguyen, J.-P. Cleuziou, S. Klyatskaya, M. Ruben and W. Wernsdorfer, Int. J. Mol. Sci., 2011, 12, 6656-6667.

64 M. Ganzhorn, S. Klyatskaya, M. Ruben and W. Wernsdorfer, Nat. Nanotechnol., 2013, 8, 165. 
65 A. I. Abd El-Mageed, M. Handayani, Z. Chen, T. Inose and T. Ogawa, Chem.-Eur. J., 2019, 25, 1941-1948.

66 M. Damjanović, Y. Horie, T. Morita, Y. Horii, K. Katoh, M. Yamashita and M. Enders, Inorg. Chem., 2015, 54, 11986-11992.

67 Y. Chen, F. Ma, X. Chen, B. Dong, K. Wang, S. Jiang, C. Wang, X. Chen, D. Qi and H. Sun, Inorg. Chem. Front., 2017, 4, 1465-1471.

68 J. Buchler, P. Hammerschmitt, I. Kaufeld and J. R. Loffer, Chem. Ber., 1991, 124, 2151-2159.

69 R. J. Donohoe, J. K. Duchowski and D. F. Bocian, J. Am. Chem. Soc., 1988, 110, 6119-6124.

70 J. W. Buchler and B. Scharbert, J. Am. Chem. Soc., 1988, 110, 4272-4276.

71 J. W. Buchler, J. Hüttermann and J. Löffler, Bull. Chem. Soc. Jpn., 1988, 61, 71-77.

72 J. K. Duchowski and D. F. Bocian, J. Am. Chem. Soc., 1990, 112, 3312-3318.

73 G. A. Spyroulias, C. P. Raptopoulou, D. de Montauzon, A. Mari, R. Poilblanc, A. Terzis and A. G. Coutsolelos, Inorg. Chem., 1999, 38, 1683-1696.

74 M. Cavallini, M. Facchini, C. Albonetti and F. Biscarini, Phys. Chem. Chem. Phys., 2008, 10, 784-793.

75 D. Yıldız, H. Ş. Şen, O. Gülseren and O. Gürlü, 2015, arXiv: 1502.00869.

76 H. Wong and C. Durkan, Phys. Rev. B: Condens. Matter Mater. Phys., 2010, 81, 045403.
77 Y. Wang, Y. Ye and K. Wu, Surf. Sci., 2006, 600, 729-734.

78 A. I. A. El-Mageed and T. Ogawa, Appl. Surf. Sci., 2018, 462, 904-912.

79 M. Bassiouk, V. A. Basiuk, E. V. Basiuk, E. Álvarez-Zauco, M. Martínez-Herrera, A. Rojas-Aguilar and I. Puente-Lee, Appl. Surf. Sci., 2013, 275, 168-177.

80 V. A. Basiuk and M. Bassiouk, J. Nanosci. Nanotechnol., 2008, 8, 259-267.

81 N. Giménez-Agulló, C. S. de Pipaón, L. Adriaenssens, M. Filibian, M. Martínez-Belmonte, E. C. Escudero-Adán, P. Carretta, P. Ballester and J. R. Galán-Mascarós, Chem.Eur. J., 2014, 20, 12817-12825.

82 M. del Carmen Giménez-López, F. Moro, A. La Torre, C. J. Gómez-García, P. D. Brown, J. Van Slageren and A. N. Khlobystov, Nat. Commun., 2011, 2, 407.

83 K. J. Sun, R. A. Wincheski and C. Park, J. Appl. Phys., 2008, 103, 023908.

84 J. P. Lu, Phys. Rev. Lett., 1995, 74, 1123.

85 H. Ajiki and T. Ando, J. Phys. Soc. Jpn., 1993, 62, 2470-2480. 86 P. Perlepe, I. Oyarzabal, K. S. Pedersen, P. Negrier, D. Mondieig, M. Rouzieres, E. A. Hillard, F. Wilhelm, A. Rogalev and E. A. Suturina, Polyhedron, 2018, 153, 248253.

87 S. Wang, J. Fu, K. Wang, M. Gao, X. Wang, Z. Wang, J. Chen and Q. Xu, Appl. Surf. Sci., 2018, 459, 208-216. 